\title{
Assessing Outcomes After Breast Surgery: Patient and Clinician Reported Outcomes
}

\author{
Jane Kilkenny ${ }^{\mathrm{a}, \mathrm{d}}$, Douglas C. Brown ${ }^{\mathrm{b}}$, Avril Gunning ${ }^{\mathrm{b}}$, Marta M. Reis ${ }^{\mathrm{b}}$, \\ E. Jane Macaskill b, c
}

\begin{abstract}
Background: Survival has significantly improved in women diagnosed with breast cancer, and as a result, it has become increasingly important to assess the psychological outcomes from the patient's perspective. Interpreting the outcome based on the opinion of the operating surgeons may not reflect the opinions of the patient. The aim of this study was to assess clinician and patient reported outcomes of breast surgery at routine follow-up.
\end{abstract}

Methods: Consecutive patients previously treated for breast cancer attending routine follow-up breast clinic over a period of 5 weeks were invited to participate. Patients were first seen by a clinician for review (four breast surgeons and one clinical nurse specialist), and cosmetic outcome was assessed using the Harris Harvard scale. Patient reported outcomes were measured using the Hopwood body image scale 10-item questionnaire.

Results: Of 105 patients, complete data were available for 84 patients. All patients were female with a median age of 65 years (range 32 - 83 years). Wide local excisions accounted for $54 \%$ of all surgeries $(n=45)$, mastectomies $26 \%(n=22)$ and mastectomy with reconstruction $20 \%(\mathrm{n}=17)$. Patients' scores ranged from 0 to 30 with a median score of $1 ; 9 \%$ of patients had a score of $>10$. Clinician rating was "excellent" for $37 \%, 34 \%$ as "very good", $22 \%$ as "good" and $5.9 \%$ as "poor". There was a weak correlation of patient scores to clinician score (Spearman rho: 0.219; 95\% CI: $0.005-0.414 ; \mathrm{P}=0.045$ ).

Conclusions: With standard breast surgery, the majority of patients seen at follow-up clinics were satisfied with their cosmetic outcome, with the most favorable outcomes in patients who had undergone

Manuscript accepted for publication May 24, 2016

${ }^{a}$ College of Medicine, Dentistry and Nursing, University of Dundee, Ninewells Hospital and Medical School, DD1 4HN, UK

bDepartment of Breast Surgery, Ninewells Hospital and Medical School, Dundee, DD1 9SY, UK

'Division of Cancer Research, Medical Research Institute, Mailbox 4, Level 7, University of Dundee, Ninewells Hospital and Medical School, DD1 9SY, UK ${ }^{\mathrm{d} C o r r e s p o n d i n g ~ A u t h o r: ~ J a n e ~ K i l k e n n y, ~ C o l l e g e ~ o f ~ M e d i c i n e, ~ D e n t i s t r y ~ a n d ~}$ Nursing, University of Dundee, Ninewells Hospital and Medical School, 21F Perth Road, Dundee, Scotland, DD1 4HU, UK. Email: jkilkenny@nhs.net

doi: http://dx.doi.org/10.14740/jcs303w breast conservation, with mastectomy and reconstruction yielding the poorest results. Patient reported outcomes are not reflected in the clinician assessment of cosmesis.

Keywords: Patient reported outcomes; PROMS; Body image; Breast surgery

\section{Introduction}

With over $75 \%$ of women now surviving for at least 10 years after breast cancer due to factors such as screening and the evolving nature of therapeutic interventions and their integration into breast cancer care, features of a chronic disease are starting to emerge $[1,2]$. As a result, assessing outcomes after treatment purely using disease recurrence and mortality is no longer sufficient [3]. There are many outcomes after breast surgery that are important to the patient such as cosmesis, the psychological impact of the surgery and the influence this has on everyday life [4]. These areas need to be explored further to give a true assessment of the outcomes after breast surgery.

The term "patient reported outcome measures" (PROMs) was introduced by the Department of Health to encompassing any measures obtained directly from the patient including areas such as health related quality of life but also including their satisfaction with care. Many instruments have been developed to measure PROs and can be divided into two broad categories: generic and specific [5]. Numerous PROMs have been developed to target patients with breast cancer; however, not all are validated, and many are ad hoc questionnaires that have not undergone any formal development or psychometric evaluation [6]. Not only should development and evaluation occur, it should occur in a specific target population. For our study, it was therefore important to identify well-developed and validated specific PROMs that are not only designed for breast cancer, but also relevant to the surgical aspects of breast cancer treatment.

\section{Materials and Methods}

A literature review was carried out to find a validated questionnaire to assess PROs. A search strategy was devised using the following key terms: breast surgery, breast oncology, breast 
Table 1. Baseline Characteristics for Patients With Complete Data $(n=84)$

\begin{tabular}{|llll}
\hline & Breast conservation $(\mathbf{n}=\mathbf{4 5})$ & Mastectomy $(\mathbf{n}=\mathbf{2 2})$ & Mastectomy with reconstruction $(\mathbf{n}=\mathbf{1 7})$ \\
\hline Median age at clinic & 63 & 70 & 51 \\
Median time since surgery (years) & 4 & 4 & 3 \\
Unilateral disease & $44(52.4 \%)$ & $18(21.4 \%)$ & $12(14.3 \%)$ \\
Bilateral disease & 0 & $3(3.6 \%)$ & $2(2.4 \%)$ \\
Bilateral surgery for symmetrizing & 0 & $1(1.2 \%)$ & $3(3.6 \%)$ \\
Axillary surgery & & & $1(1.2 \%)$ \\
$\quad$ None & $11(13.1 \%)$ & 0 & $9(10.7 \%)$ \\
$\quad$ Sentinel node & $22(26.2 \%)$ & $6(7.1 \%)$ & $2(2.4 \%)$ \\
$\quad$ Axillary sample & $7(8.3 \%)$ & $2(2.4 \%)$ & $9(10.7 \%)$ \\
$\quad$ Axillary clearance & $5(6 \%)$ & $18(21.4 \%)$ & $8(9.5 \%)$ \\
Chemotherapy & $9(10.7 \%)$ & $7(8.3 \%)$ & $6(7.1 \%)$ \\
Radiotherapy & $41(48.8 \%)$ & $10(11.9 \%)$ & $8(9.5 \%)$ \\
Hormonal therapy & $25(29.8 \%)$ & $14(16.7 \%)$ & \\
\hline
\end{tabular}

cancer, breast reconstruction, mastectomy, patient reported outcomes, quality of life, health related quality of life, patient satisfaction, body image, questionnaire, validated tool, and validated instrument. The following databases were examined: Medline, PubMed, and Ovid Evidence Based Medicine databases. All non-English papers were excluded. All instruments included in this review were identified as PROMs measuring breast cancer related quality of life. A shortlist of potential questionnaires was presented to a multidisciplinary panel prior to final selection. Ethical approval was not sought as part of this study due to the collection of PROs being a service improvement measure.

All female patients previously treated for cancer attending a routine follow-up clinic over a period of 5 weeks were invited to participate. All women had undergone surgical intervention for breast cancer. Patients were invited to take part in the study at the end of their consultation. The finalized PROM was a two-part questionnaire. The first part was a clinician reported cosmesis scale developed by Harris (Harvard scale) [7, 8] filled out once clinical review was completed (Supplementary 1, www.currentsurgery.org). The second part was the Hopwood body image scale (BIS) questionnaire (Supplementary 2, www.currentsurgery.org) [9] which was given to patients who were then shown to a designated area to complete the questionnaire, and place in a box anonymously.

Patient demographics and treatment details were collected from the patient files by the clinician after seeing each patient. Cosmesis was assessed by the clinician for each patient using the Harris Harvard cosmesis scale which rated cosmesis on a scale from "excellent" to "poor". BIS score out of 30 was calculated for each patient based upon their responses. Responses were paired for each patient by an anonymized non-identifiable study number.

\section{Statistical analysis}

Statistical analysis was carried out using the VassarStats ${ }^{\complement}$ on- line statistical computation package (http://faculty.vassar.edu/ lowry/VassarStats.html). Analysis of differences between median scores for each subgroup was performed by Mann-Whitney test of non-parametric difference in independent samples. Rank correlation between clinician and patient reported outcomes was performed using Microsoft Excel 2013. Statistical results indicating a less than $5 \%$ probability of the results due to chance $(\mathrm{P}<0.05)$ were considered significant.

\section{Results}

\section{Literature review and selection of PROM}

The review of the literature found nine potential PROMs suitable for the study. These were European Organization for Research and Treatment of Cancer Quality of Life questionnaire - breast cancer module - EORTC QLQ-BR23 [10]; Functional Assessment of Cancer Therapy-Breast Cancer (FACT-B) [11]; The Breast Q (3) [3]; Michigan Breast Reconstruction Outcomes Study (MBROS) [12, 13]; Breast Cancer Treatment

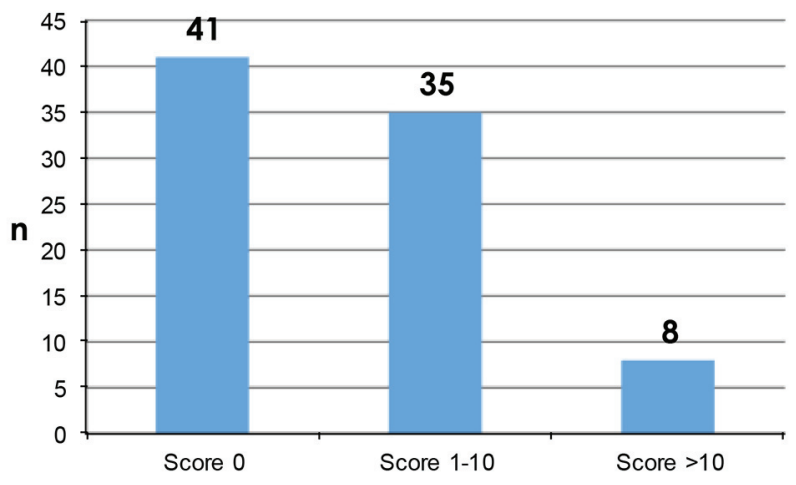

Figure 1. Patient reported Hopwood BIS scores. 


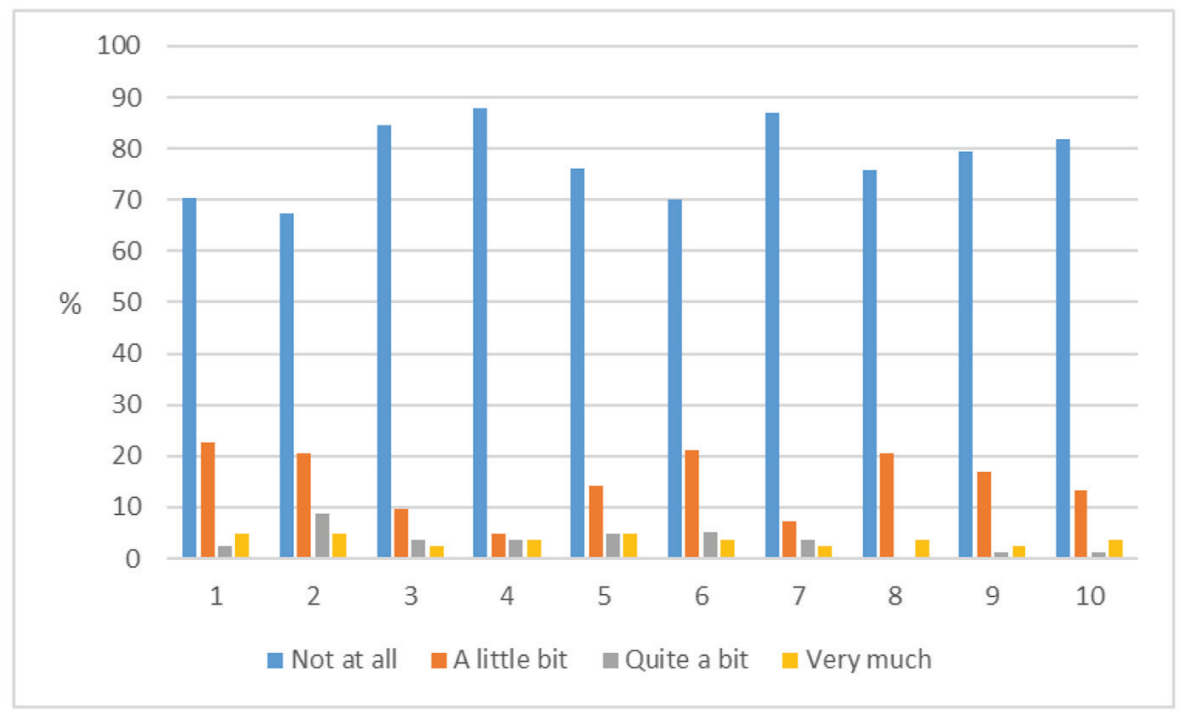

Figure 2. Responses to Hopwood score for each of the 10 questions (1 - 10, Supplementary 1, www.currentsurgery.org).

Outcome Scale (BCTOS) [14]; Mastectomy Attitude Scale (MAS) [15]; Hopwood Body Image Scale [9]; Body Image After Breast Cancer Questionnaire (BIBCQ) [16]; Polivy Body Image Scale (PBIS) [17].

These were then assessed by the following criteria: clinically validation; surgery specific; incorporating psychological and body image components; short completion time; patient involvement during development of the tool. After discussion with a multidisciplinary panel, this Hopwood BIS was selected for use in our study. It is a 10-item questionnaire developed to assess body image changes in patients with cancer. It is clinically validated and takes on average 3 min to complete [9]. This instrument was developed with extensive patient interviews.

\section{Data collection}

Of the 105 patients seen in clinic over the 5 weeks, 84 patients were included in the study. Nineteen patients were excluded due to either incomplete core data $(n=17)$, patients deemed not suitable $(n=3)$ or patient declined $(n=1)$. All patients involved in the study were female with a median age of 65 years (range 32 - 83 years). Baseline demographic and clinical information recorded by the clinician is summarized in Table 1. Most $(88 \%)$ of patients had unilateral disease, $5.9 \%$ had bilateral disease, and $4.7 \%$ underwent bilateral surgery for symmetrising.

PROs were measured using the Hopwood BIS (Supplementary 1, www.currentsurgery.org). Patient responses were scored on an ordinal scale using the numbers $0-3$ to represent four possible opinions ranging from "not at all" to "very much" with a total possible score of 30 . Higher scores were indicative of greater dissatisfaction with their body image. A high proportion of patients have had no impact on their body image from their surgery while a minority of patients reported that their surgery has had quite a bit or very much impact on their body image (Figs. 1 and 2).

Results were analyzed by the type of surgery, time elapsed since surgery, age at time of assessment and estimation of disease severity comparing those patients that had both chemotherapy and axillary clearance versus neither (Table 2).

While there was no difference in age or time elapsed since surgery, there were significant differences in overall scores for type of surgery, with better results for WLE than mastectomy with or without reconstruction $(\mathrm{P}=0.01)$. Patients who had estimated poorer prognostic disease (chemotherapy and axillary clearance) had poorer scores than those with estimated good prognostic disease (neither chemotherapy nor axillary clearance) $(\mathrm{P}=0.003)$.

Clinician reported outcomes as assessed by Harris Harvard scale are shown in Figure 3, with poor results reported in five patients $(5.9 \%)$.

These data were analyzed alongside the BIS scores to show whether or not the cosmetic outcome as viewed by the clinician is correlated to the PROs. The results shown in Figure 4 demonstrate weak correlation of patient scores to clinician score (Spearman rho: 0.219; (95\% CI: 0.005 - 0.414; $\mathrm{P}=$ $0.045)$.

\section{Discussion}

This study has allowed us to gain valuable insight into the patient's perspective of their body image after surgery. Just under half of patients have had no impact on their body image from their breast surgery. There were, however, a minority of patients who reported that their body image has been very much affected by their surgery. In order to fully understand why some patients have more body image concerns, it is necessary to consider BIS scores in the context of their age, time since surgery and the type of surgery they underwent as well as look- 
Table 2. Patient Reported Body Image Scores by Surgery Type, Age and Time Since Surgery

\begin{tabular}{|c|c|c|c|}
\hline Variable & Number of patients & Patient score, median (range) & P-value (Mann-Whitney) \\
\hline \multicolumn{4}{|l|}{ Surgery type } \\
\hline Wide local excision (WLE) & 45 & $0(0-20)$ & WLE vs. $\mathrm{M}, \mathrm{P}=0.01$ \\
\hline Mastectomy with reconstruction (MR) & 17 & $4(0-24)$ & WLE vs. MR, $\mathrm{P}=0.01$ \\
\hline \multicolumn{4}{|l|}{ Current age } \\
\hline \multicolumn{4}{|l|}{ Years since surgery } \\
\hline$<2$ years $(1-2$ years $)$ & 23 & $0(0-20)$ & \\
\hline$\geq 2$ years $(2-14$ years $)$ & 61 & $0.5(0-30)$ & $\mathrm{P}=0.77(\mathrm{NS})$ \\
\hline \multicolumn{4}{|l|}{ Estimated extent of disease } \\
\hline
\end{tabular}

NS: not statistically significant.

ing at responses to the specific questions.

Many studies have shown that the type of surgical procedure undergone has had an effect on post-surgical body image $[18,19]$. A similar study carried out in the UK showed that a significantly better body image was observed in patients after breast conservation compared with mastectomy with or without reconstruction [18]. Our findings confirm better body image scores after breast conservation, but with poorest results in those having had mastectomy and reconstruction (MR) (Table 2). There are several possible reasons for this poorer outcome, but in the absence of pre-surgical body image scoring, it is difficult to know what effect their pre-treatment personalities and their psychological background has had on their body image adjustment. It is probable that the women who chose to have a reconstruction had more body image concerns beforehand than patients who had a mastectomy alone. The cohort of patients that underwent MR was significantly younger (median age 51 years) than the group that had a mastectomy alone (median age 70 years) which could also be contributing to a poorer BIS score.

Immediate reconstruction is offered to all women undergoing mastectomy in our department, including women with poorer prognosis tumours. We were not able to collect specific pathology information for this study due to the anonymous data collection, but can estimate those with poorer prognostic disease as those who received chemotherapy and had axillary clearance, which is only performed in our unit for node positive disease. In the above Japanese study, there was a higher

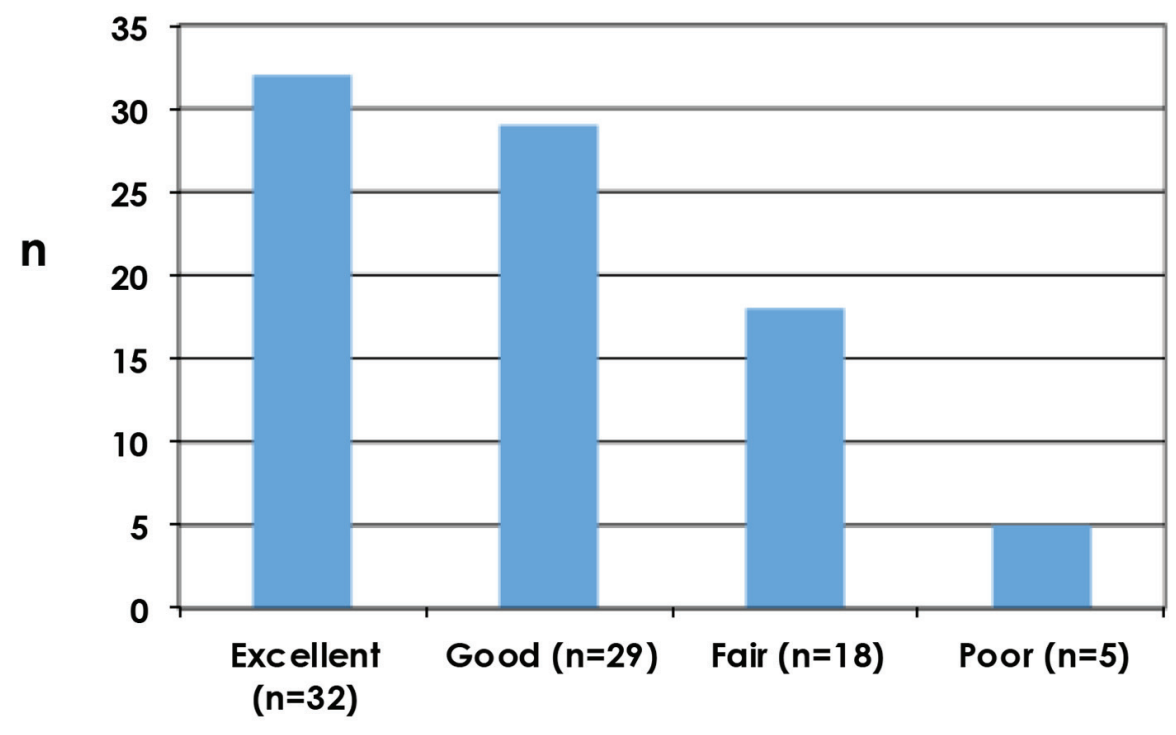

Figure 3. Cosmetic outcomes as assessed by the clinician using Harvard scale. 


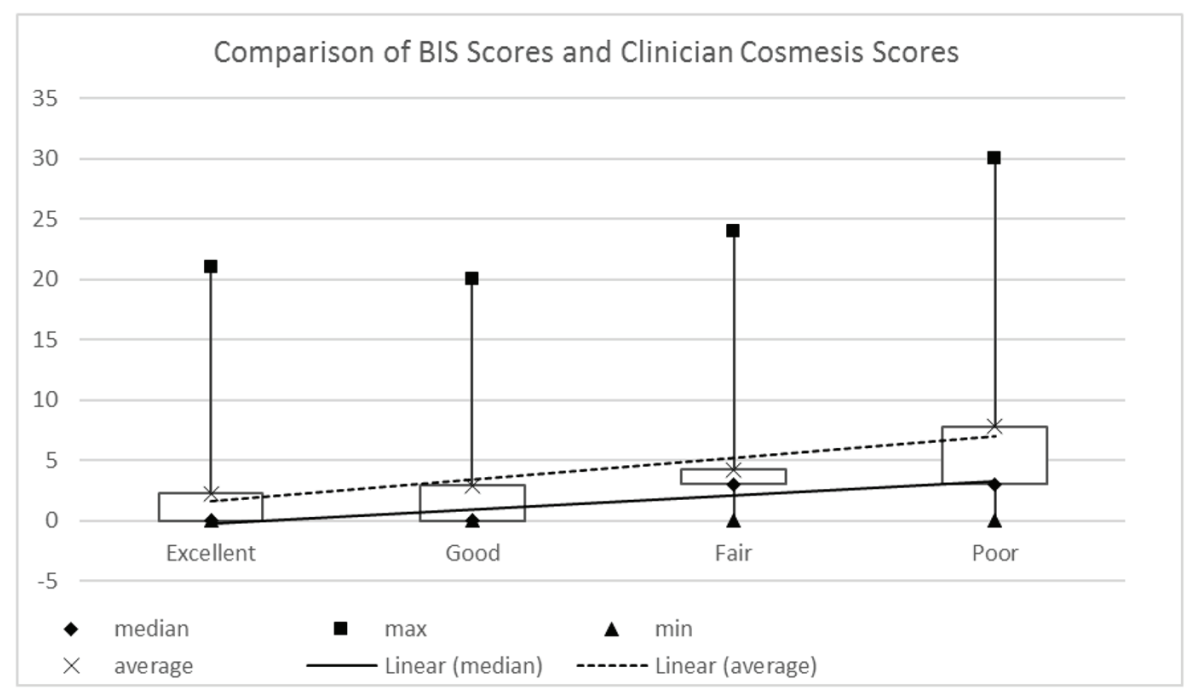

Figure 4. Patient reported BIS scores and clinician assessed Harvard scores (Spearman rho: 0.219; $95 \%$ Cl: 0.005 - 0.414 ; P $=0.045$ ).

proportion of women in the mastectomy only group who had chemotherapy and axillary dissection, compared with MR and WLE [19]. These differences in disease prognosis may have influenced patient perspective on body image. In our study, there were few patients in the breast conservation group $(6 \%)$ with estimated poor prognostic disease, but far higher numbers in the mastectomy only group (31\%) and in the MR group (41\%), allowing better comparison between the two mastectomy groups than in the Japanese study where rates for WLE and MR were similar. Both studies demonstrate the influence of disease prognosis upon body image, and this warrants further investigation.

Studies have previously shown a significant relationship between body image concerns and time elapsed after surgery, with a worsening of body image in the first few months postoperatively $[9,17,20,21]$. Our data were analyzed for results for less than 2 years postoperative and greater than 2 years postoperative, but there was no difference between the two groups.

A study by Collins et al interviewed a cohort of patients at 4 - 6 weeks, 6 months, 12 months and 24 months after surgery to assess the effects of breast surgery on body image over time [22]. They found that after 6 months, patients who underwent MR had a poorer body image than the mastectomy group but after 24 months, the body image problems did not differ significantly [22]. Similar findings were observed in our study. Although the numbers are small, our data suggest that that MR had the poorest BIS score in the first 2 years following surgery but after 2 years, MR and mastectomy had a similar score. Patients who had breast conservation had a median BIS score of zero in both timeframes. One of the limitations of this study's "snapshot" assessment of body image is the inability to detect these changes in an individual over time. The use of a tool at specific time intervals in a patient's treatment journey would be valuable, but was not within the constraints of our study aims.
The results of individual questions within the study were analyzed by surgery performed, and showed that patients having breast conservation had better scores in all individual questions than the mastectomy and MR groups. Our data have shown that patients having reconstruction after mastectomy were more likely to feel much more self-conscious in the MR group compared to the mastectomy group but when asked if they avoided people because of how they felt about their appearance, both groups faired similarly. Younger women tend to experience a higher societal influence on their body image which could be a contributory factor in why the MR group, which has a much younger median age group, felt more selfconscious than the mastectomy group [23].

The MR group overall felt both less physically and sexually attractive as a result of their disease/treatment compared to the mastectomy group and a higher proportion also reported that they felt less feminine. It is clear that the MR group reported consistently poorer outcomes and possible reasons for this have been previously mentioned but the causes have not been fully established due to the involvement of other variables that have not been addressed in this study.

Our service currently does not routinely assess PROs and solely depends on cosmetic outcome as viewed by the clinician to evaluate and improve their service, or individual patients requesting referral for adjustment surgery. This study has shown that there is only a weak correlation between PROs and clinician reported outcomes. This reinforces the need to assess PROs in the future so that outcomes other than just cosmesis can be taken into account.

\section{Conclusion}

This study has shown that breast conservation results in better body image than mastectomy or reconstruction. It has found that poorer body image scores were seen in patients who had 
more extensive cancer treatment and patients that had undergone reconstruction. PROs should be routinely assessed in patients before and after breast surgery to identify patients at risk of body image issues, in particular those undergoing breast reconstruction. The process of a psychological "screen" before surgery could allow for increased input or support to be offered to patients who may be identified as at risk psychologically after their surgery. This would allow the service to be altered to support these patients so that they can achieve the best outcome possible from their surgery.

\section{References}

1. Coleman M. Statistics and outlook for breast cancer: Cancer Research UK; 2012 [updated 10/05/201213/04/2014]. Available from: http://www.cancerresearchuk.org/cancerinfo/cancerstats/types/breast/survival/.

2. Kanatas A, Velikova G, Roe B, Horgan K, Ghazali N, Shaw RJ, Rogers SN. Patient-reported outcomes in breast oncology: a review of validated outcome instruments. Tumori. 2012;98(6):678-688.

3. Pusic AL, Klassen AF, Scott AM, Klok JA, Cordeiro PG, Cano SJ. Development of a new patient-reported outcome measure for breast surgery: the BREAST-Q. Plast Reconstr Surg. 2009;124(2):345-353.

4. Potter S, Harcourt D, Cawthorn S, Warr R, Mills N, Havercroft D, Blazeby J. Assessment of cosmesis after breast reconstruction surgery: a systematic review. Ann Surg Oncol. 2011;18(3):813-823.

5. Morley D. A structured review of patient reported outcome measures for patients undergoing cosmetic surgical procedures: Department of Public Health, University of Oxford 2013 [13/04/2014]. Available from: http://phi.uhce.ox.ac.uk/pdf/Cosmetic\%20Surgery\%20 PROMs\%20Review2013.pdf.

6. Chen CM, Cano SJ, Klassen AF, King T, McCarthy C, Cordeiro PG, Morrow M, et al. Measuring quality of life in oncologic breast surgery: a systematic review of patientreported outcome measures. Breast J. 2010;16(6):587597.

7. Harris JR, Levene MB, Svensson G, Hellman S. Analysis of cosmetic results following primary radiation therapy for stages I and II carcinoma of the breast. Int J Radiat Oncol Biol Phys. 1979;5(2):257-261.

8. Cardoso MJ, Cardoso JS, Vrieling C, Macmillan D, Rainsbury D, Heil J, Hau E, et al. Recommendations for the aesthetic evaluation of breast cancer conservative treatment. Breast Cancer Res Treat. 2012;135(3):629637.

9. Hopwood P, Fletcher I, Lee A, Al Ghazal S. A body image scale for use with cancer patients. Eur J Cancer. 2001;37(2):189-197.

10. Sprangers MA, Groenvold M, Arraras JI, Franklin J, te Velde A, Muller M, Franzini L, et al. The European Organization for Research and Treatment of Cancer breast cancer-specific quality-of-life questionnaire module: first results from a three-country field study. J Clin Oncol.
1996;14(10):2756-2768.

11. Brady MJ, Cella DF, Mo F, Bonomi AE, Tulsky DS, Lloyd SR, Deasy S, et al. Reliability and validity of the Functional Assessment of Cancer Therapy-Breast quality-of-life instrument. J Clin Oncol. 1997;15(3):974-986.

12. Alderman AK, Wilkins EG, Lowery JC, Kim M, Davis JA. Determinants of patient satisfaction in postmastectomy breast reconstruction. Plast Reconstr Surg. 2000;106(4):769-776.

13. Wilkins EG, Cederna PS, Lowery JC, Davis JA, Kim HM, Roth RS, Goldfarb S, et al. Prospective analysis of psychosocial outcomes in breast reconstruction: oneyear postoperative results from the Michigan Breast Reconstruction Outcome Study. Plast Reconstr Surg. 2000;106(5):1014-1025; discussion 1026-1017.

14. Stanton AL, Krishnan L, Collins CA. Form or function? Part 1. Subjective cosmetic and functional correlates of quality of life in women treated with breastconserving surgical procedures and radiotherapy. Cancer. 2001;91(12):2273-2281.

15. Reaby LL, Hort LK, Vandervord J. Body image, self-concept, and self-esteem in women who had a mastectomy and either wore an external breast prosthesis or had breast reconstruction and women who had not experienced mastectomy. Health Care Women Int. 1994;15(5):361-375.

16. Baxter NN, Goodwin PJ, McLeod RS, Dion R, Devins G, Bombardier C. Reliability and validity of the body image after breast cancer questionnaire. Breast $\mathrm{J}$. 2006;12(3):221-232.

17. Polivy J. Psychological effects of mastectomy on a woman's feminine self-concept. J Nerv Ment Dis. 1977;164(2):77-87.

18. Al-Ghazal SK, Fallowfield L, Blamey RW. Comparison of psychological aspects and patient satisfaction following breast conserving surgery, simple mastectomy and breast reconstruction. Eur J Cancer. 2000;36(15):19381943.

19. Sun Y, Kim SW, Heo CY, Kim D, Hwang Y, Yom CK, Kang E. Comparison of quality of life based on surgical technique in patients with breast cancer. Jpn J Clin Oncol. 2014;44(1):22-27.

20. Ganz PA, Coscarelli A, Fred C, Kahn B, Polinsky ML, Petersen L. Breast cancer survivors: psychosocial concerns and quality of life. Breast Cancer Res Treat. 1996;38(2):183-199.

21. Sneeuw KC, Aaronson NK, Yarnold JR, Broderick M, Regan J, Ross G, Goddard A. Cosmetic and functional outcomes of breast conserving treatment for early stage breast cancer. 1. Comparison of patients' ratings, observers' ratings, and objective assessments. Radiother Oncol. 1992;25(3):153-159.

22. Collins KK, Liu Y, Schootman M, Aft R, Yan Y, Dean G, Eilers M, et al. Effects of breast cancer surgery and surgical side effects on body image over time. Breast Cancer Res Treat. 2011;126(1):167-176.

23. Pruis TA, Janowsky JS. Assessment of body image in younger and older women. J Gen Psychol. 2010;137(3):225-238. 